\title{
The Role of MicroRNAs in Vitiligo: Regulators and Therapeutic Targets
}

\author{
Lili Li
}

Department of Dermatology, People's Hospital of Guangxi Zhuang Autonomous Region, Nanning, China

Vitiligo is an acquired skin disorder clinically characterized by the progressive appearance of white maculae due to a loss of functioning epidermal melanocytes. Studies have shown that microRNAs (miRNAs) modulate cellular differentiation, proliferation and apoptosis, including immune cell and melanocyte development and functions. The role of miRNAs in the pathogenesis of several immune-related diseases has been explored. Novel approaches to target miRNAs have recently emerged allowing modulation of miRNAs levels in diverse pathological processes, thus making them promising targets for molecular-based diagnostics and therapy. Here, we report the present status of research on miRNAs expression and functional alterations in vitiligo, in order to more fully understand the role of these molecules in vitiligo pathology. (Ann Dermatol 32(6) $441 \sim 451,2020$ )

\section{-Keywords-}

Melanocytes, MicroRNAs, Pathogenesis, Therapeutics, Vitiligo

\section{INTRODUCTION}

Vitiligo is a common skin depigmenting disorder clinically

Received October 22, 2019, Revised May 12, 2020, Accepted for publication May 15, 2020

Corresponding author: Lili Li, Department of Dermatology, People's Hospital of Guangxi Zhuang Autonomous Region, 6 Taoyuan Road, Nanning 530021, China. Tel: 86-18777190925, Fax: 86-771-2186106, E-mail: 18777190925@ 163.com

ORCID: https://orcid.org/0000-0002-9662-287X

This is an Open Access article distributed under the terms of the Creative Commons Attribution Non-Commercial License (http://creativecommons. org/licenses/by-nc/4.0) which permits unrestricted non-commercial use, distribution, and reproduction in any medium, provided the original work is properly cited.

Copyright $(c)$ The Korean Dermatological Association and The Korean Society for Investigative Dermatology characterized by the progressive appearance of white spots and patches due to the destruction of epidermal melanocytes ${ }^{1,2}$. The onset and progression of vitiligo are thought to be driven by multiple inherited genes and environmental triggers, with a complex pathogenesis. The precise molecular mechanism of vitiligo pathogenesis has remained elusive.

MicroRNAs (miRNAs) are highly conserved, noncoding small RNAs that play an important role in diverse physiological and developmental processes in humans. Several studies have demonstrated that miRNAs acting as regulatory factors are involved in various important aspects of the pathological process of vitiligo, such as the growth, differentiation and apoptosis of melanocytes and the immune response in vitiligo ${ }^{3,4}$.

Here, we report the present status of research on miRNA expression and functional alterations in vitiligo to more fully understand the role of these molecules in vitiligo pathology.

\section{PATHOGENESIS OF VITILIGO}

\section{Pathogenetic mechanisms}

Currently, many theories have been proposed to explain the mechanisms of pigmentation loss, including autoimmune dysfunction, neurotoxic factor destruction, and genetic factors, and so on (Fig. 1).

\section{1) Genetic factors associated with vitiligo}

Genome-wide association studies have discovered approximately 50 different genetic loci that contribute to vitiligo risk $^{5}$. A large fraction of these genes encode proteins involved in immune regulation. Some genes also play roles in cellular apoptosis, and others are involved in regulating the functions of melanocytes. As vitiligo is a polygenic disease, several candidate genes that are involved in the reg- 


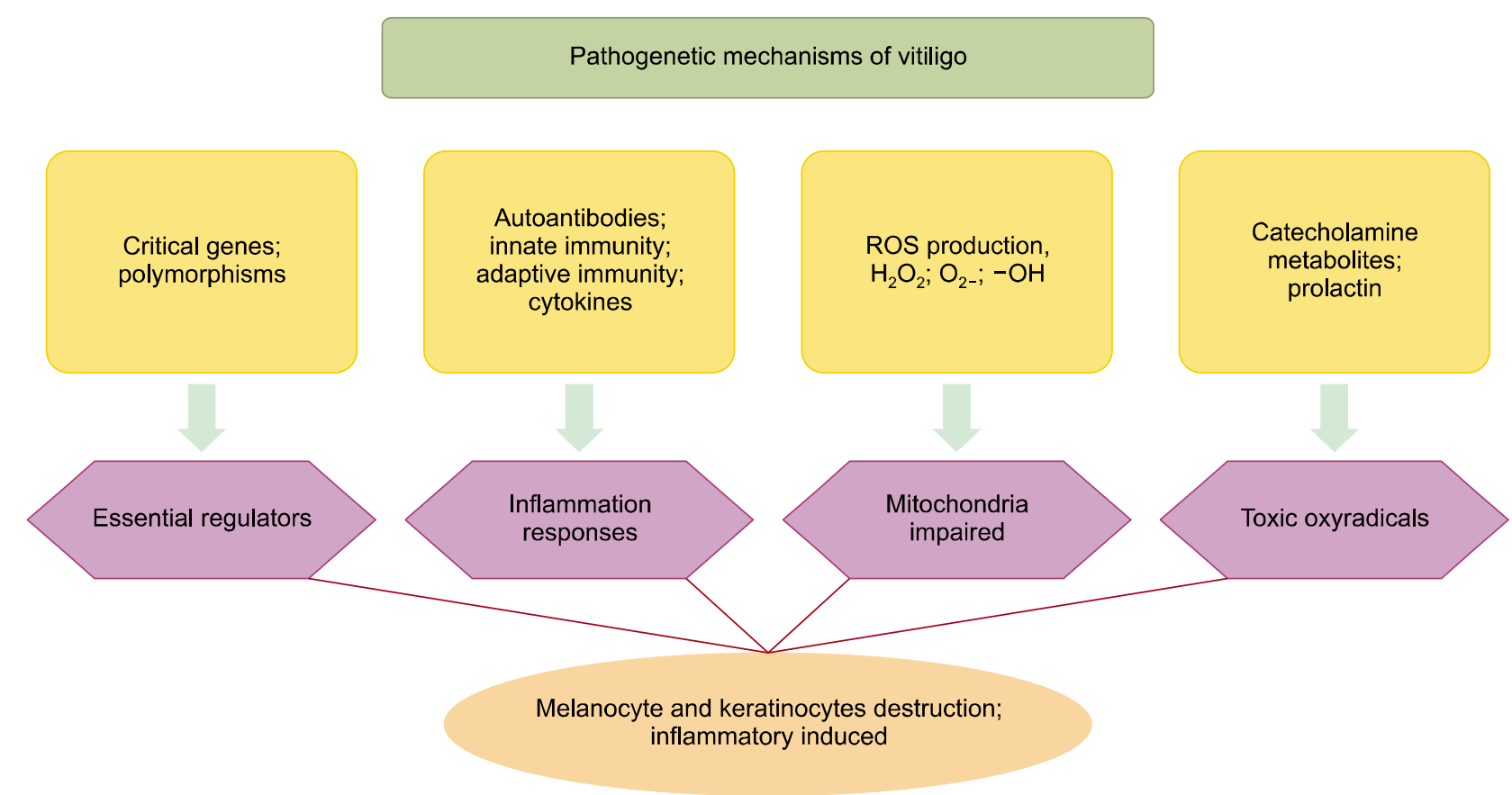

Fig. 1. The suggested pathogenetic mechanisms of vitiligo. Some theories have been proposed to explain the pathological mechanism of vitiligo, including genetic factors, autoimmune dysfunction, oxidative damage and neurotoxic factor destruction. ROS: reactive oxygen species, $\mathrm{OH}$ : hydroxide.

ulation of immunity, such as major histocompatibility complex (MHC), cytotoxic T lymphocyte antigen-4 (CTLA4) and interleukin-2 receptor $\mathrm{A}(I L 2 R A)$, have been tested for genetic association with vitiligo ${ }^{6-8}$.

Recently, the role of genetic polymorphisms and noncoding RNAs in the pathogenesis of vitiligo has been explored. Genetic polymorphisms include single-nucleotide polymorphisms (SNPs), nucleotide insertions, deletions, inversions and chromosomal translocations ${ }^{9,10}$. Many studies have identified that several SNPs, such as the NRF2 rs35652124, PSMB8 rs2071464 and inducible nitric oxide synthase (NOS2) gene polymorphism, were all associated with increased susceptibility to vitiligo ${ }^{11,12}$. Most SNPs could target promoter regions of genes that play critical regulatory roles in vitiligo pathogenesis.

The decreased expression of epithelial cadherin (E-cadherin) and discoidin domain receptor tyrosine kinase 1 (DDR1) have been implicated as aggravating factors in the loss of melanocytes in vitiligo ${ }^{13,14}$. Recent studies have shown that SNPs in genes encoding these two proteins, $\mathrm{CDH} 1$ and DDR1, are correlated with susceptibility to vitiligo. As Almasi-Nasrabadi et al. ${ }^{15}$ demonstrated the $\mathrm{CDH} 1 \mathrm{CC}$ genotype and DDR1 CC genotype were found to be significantly associated with the risk of developing vitiligo.

GPX1 is a major intracellular antioxidant enzyme that has been shown to decrease GPX1 activity in patients with vitiligo. Mansuri et al. ${ }^{16}$ confirmed that the variations in the Arg5Pro and Leu6Pro SNPs in GPX1 induced decreased GPX1 activity in patients, suggesting the crucial role of genetic factors in vitiligo pathogenesis.

Further functional studies of these SNPs may contribute to elucidating the pathogenesis of vitiligo.

\section{2) Immune pathogenesis of vitiligo}

Vitiligo is an immune disease. Current research indicates that autoantibodies, innate immunity, adaptive immunity and cytokines are involved in the regulation of melanocyte destruction or apoptosis, which is the main mechanism leading to the pathogenesis of vitiligo ${ }^{17}$.

Autoreactive T cells were implicated in killing melanocytes in vitiligo skin, attributed to the discovery of labeled $T$ cells isolated from vitiligo lesions infiltrating the normal skin. Studies have demonstrated that $\mathrm{CD}^{+} \mathrm{T}$ cells are key effectors that drive melanocyte destruction in patients with vitiligo and are involved in the recruitment of these pathogenic cells to skin tissue. Depletion of $\mathrm{CD}^{+}{ }^{+} \mathrm{T}$ cells prevented melanocyte destruction, whereas enrichment for these cells enhanced it, supporting the key role of $\mathrm{CD}^{+} \mathrm{T}$ cells in vitiligo ${ }^{18-20}$.

Autoantibodies are another factor that participate in regulating melanocyte destruction. Anti-melanocyte antibody immunoglobulin $G$ mediates the production of human leukocyte antigen-DR (HLA-DR), intercellular cell adhesion molecule-1 (ICAM-1) and interleukin (IL)-8 which play 
important roles in inflammation responses and melanocyte destruction in vitiligo ${ }^{21-24}$.

Antigenic proteins in vitiligo have been identified, and include glycoprotein 100 (gp100), melanoma antigen recognized by $\mathrm{T}$ cells (MART-1), tyrosinase, and tyrosinase related proteins 1 and 2, all of which are specific melanocyte antigens $^{25-27}$. In vitiligo, antibodies are produced against the antigens of melanocytes and released during the process of active melanocyte destruction by cellular immunity. A more recent study found that keratinocytes are the major source of chemokine production during vitiligo and that disrupting interferon (IFN)- $\gamma$ signaling only in keratinocytes mitigated disease in mice ${ }^{28}$. This suggests that targeting IFN- $\gamma$ signaling in keratinocytes could also be an effective treatment strategy.

\section{3) The oxidative damage}

The oxidative stress hypothesis indicated an imbalanced redox state in vitiliginous skin. This results in the dramatic production of reactive oxygen species (ROS), leading to melanocyte destruction and creating depigmented macules $^{29}$. Various cytokines lead to ROS overproduction in the melanocytes of vitiligo, such as TNF- $\alpha$, basic fibroblast growth factor (bFGF), IL-6, and IL- $1^{30}$. These factors contribute to the accumulation of ROS in melanocytes, finally leading to the damage of melanocytes and the production of autoantigens through the process of apoptosis, endoplasmic reticulum stress or autophagy ${ }^{31}$.

Mitochondria are the crucial cytoplasmic organelles responsible for the generation of energy to maintain cellular homeostasis. The dysregulation of mitochondrial function is involved in several diseases, especially oxidative stress-associated tissue and cell damage ${ }^{32}$. Several studies have shown that mitochondrial dysregulation is associated with oxidative stress-induced melanocyte destruction in vitiligo ${ }^{33}$.

\section{4) Neurochemical factor impairment}

The neural hypothesis of vitiligo etiology is supported by several findings that different neurochemical mediators secreted by the nerve endings are toxic to melanocytes, leading to their destruction. Neural factors as well as emotional and/or stressful events seem to play a pivotal role in vitiligo onset or exacerbation ${ }^{34}$.

The levels of some catecholamine metabolites are significantly higher in patients with vitiligo ${ }^{34}$. One mechanism by which catecholamine metabolites impact melanocytes is through the direct cytotoxic action of catecholamines and their o-diphenol catabolites. Another influencing factor is that skin and mucosa arterioles possess receptors that may cause severe vasoconstriction when activated by catecholamine discharge, leading to epidermal and der- mal hypoxia with hyperproduction of toxic oxyradicals generated by different pathways ${ }^{35}$.

Prolactin, as a hormone secreted by lactotroph cells in the pituitary gland, has recently been shown to have additional functions in skin biology and hair follicle growth. It is known as an immunomodulatory factor for lymphocytes with the potential to stimulate immune responses at many levels. Recently, prolactin has been implicated in the function of the dendritic cells in vitiligo and other skin diseases $^{36}$.

The onset of vitiligo may be caused by individuals with genetic background under the influence of various internal and external environmental factors, which induce immune function, oxidative stress, neurohumoral, melanocyte apoptosis and loss, further resulting in melanocyte destruction, melanin production or pigmentation dysfunction eventually leads to melanin loss.

\section{MiRNA IN VITILIGO}

MiRNAs are a class of small noncoding RNA molecules approximately 22 nucleotides in length that regulate gene expression by targeting mRNA. MiRNAs can bind with partial complementarity to sequences in the $3^{\prime}$ untranslated region ( $3^{\prime}$-UTR) of target mRNAs for the regulation of gene expression.

A growing number of events have shown that miRNAs are crucial for skin homeostatic maintenance (Table 1$)^{37-51}$.

\section{Immune-related miRNAs in vitiligo}

Cellular and humoral immunity have been confirmed to be of etiological significance in the pathogenesis of vitiligo. Recently, studies reported that some miRNAs have been implicated in the immune response ${ }^{3}$. These miRNAs regulate a broad range of biological processes, and play a potential role in human cutaneous $\mathrm{T}$ lymphocytes in the mechanism of immune imbalance in vitiligo.

A study showed that miR-133b expression increased in lesional skin from NSV, which was identified to be located directly upstream of the IL17A or IL17F locus. IL-17A/F and IL-22 secretion from Th17 cells could stimulate epithelial cells to produce chemokines and cytokines in vitiligo. This suggests that miR-133b plays an important role in the pathogenesis of vitiligo ${ }^{37}$.

In addition, upregulation of miR-224-3p and miR-4712-3p and downregulation of miR-3940-5p induced by the immune modulator thymosin $\alpha 1$ ( $\mathrm{T} \alpha 1$ ) were observed in NSV patients ${ }^{38}$. Wang et al. ${ }^{52}$ demonstrated that inhibition of miR-3940-5p promotes T-cell activity by targeting the cytokine receptor IL-2R, suggesting that miR-3940-5p might become potential therapeutic target in the treatment of 
Table 1. The role of microRNAs (miRNAs) in vitiligo

\begin{tabular}{|c|c|c|c|c|c|c|}
\hline $\begin{array}{l}\text { Functions in } \\
\text { vitiligo }\end{array}$ & MiRNA & Expression level & Source & $\begin{array}{l}\text { Target } \\
\text { gene }\end{array}$ & $\begin{array}{l}\text { Detection } \\
\text { methodology }\end{array}$ & Reference \\
\hline \multirow[t]{3}{*}{$\begin{array}{l}\text { Immune } \\
\text { response }\end{array}$} & miR-133b & Upregulated & $\begin{array}{l}\text { Lesional skin of } \\
\text { NSV patients }\end{array}$ & IL17a/f & Low density array & 37 \\
\hline & $\begin{array}{l}\text { miR-224-3p, } \\
\text { miR-4712-3p }\end{array}$ & Upregulated & PBMC of NSV patients & $\mathrm{T} \alpha 1$ & miRNA array & 38 \\
\hline & miR-3940-5p & Downregulated & PBMC of NSV patients & $\mathrm{T} \alpha 1$ & miRNA array & 38 \\
\hline \multirow[t]{8}{*}{$\begin{array}{l}\text { Melanocyte } \\
\text { function }\end{array}$} & $\begin{array}{l}\text { hsa-miR-1225-3p, } \\
\text { hsa-miR-634, } \\
\text { hsa-miR-197, } \\
\text { hsa-miR-766, } \\
\text { hsa-miR-574-5p, } \\
\text { hsa-miR-328 }\end{array}$ & Upregulated & $\begin{array}{l}\text { MITF knocked } \\
\text { down melanocytes } \\
\text { model }\end{array}$ & MITF & miRNA microarray & 39 \\
\hline & $\begin{array}{l}\text { hsa-miR-720, } \\
\text { hsa-miR-1308 }\end{array}$ & Downregulated & $\begin{array}{l}\text { MITF knocked } \\
\text { down melanocytes } \\
\text { model }\end{array}$ & MITF & miRNA microarray & 39 \\
\hline & miR-434-5p & Upregulated & $\begin{array}{l}\text { The skin tissue of } \\
\text { Patched albino (white) } \\
\text { skins of } \\
\text { melanin-knockdown } \\
\text { mice }\end{array}$ & TYR/HYAL & Microarray analyses & 40 \\
\hline & miR-330-5p & Upregulated & $\begin{array}{l}\text { Human melanoma cell } \\
\text { model }\end{array}$ & TYR & $\begin{array}{l}\text { In silico prediction } \\
\text { (mirDIP) }\end{array}$ & 41 \\
\hline & miR-137, miR-148 & - & $\begin{array}{l}\text { Melanocytes and } \\
\text { melanoma cells }\end{array}$ & MITF & PCR & 42 \\
\hline & miR-145 & Downregulated & $\begin{array}{l}\text { Murine melan- a } \\
\text { melanocytes model and } \\
\text { the lesional skin of } \\
\text { vitiligo patients }\end{array}$ & TYR, MITF & RT-qPCR & 43,44 \\
\hline & miR-155 & Upregulated & $\begin{array}{l}\text { Lesional skin of } \\
\text { vitiligo patients }\end{array}$ & $\begin{array}{l}\text { SOCS1/ } \\
\text { TRP1 }\end{array}$ & RT-qPCR & 44 \\
\hline & miR-211 & Downregulated & $\begin{array}{l}\text { Mouse melanocytes } \\
\text { model }\end{array}$ & TGFBR2 & RT-qPCR & 45 \\
\hline \multirow[t]{4}{*}{ Oxidative stress } & miR-135a, miR-9 & Upregulated & $\begin{array}{l}\text { Lesional skin of } \\
\text { NSV patients }\end{array}$ & SIRT1 & Low density array & 37 \\
\hline & miR-183 & Upregulated & $\begin{array}{l}\text { Lesional skin of } \\
\text { NSV patients }\end{array}$ & $\mathrm{HO} 1$ & Low density array & 37 \\
\hline & miR-1 & Upregulated & Skin of vitiligo patients & $\begin{array}{l}\text { HSP60/ } \\
\text { HSP70 }\end{array}$ & Low density array & 46 \\
\hline & miR-25 & Upregulated & $\begin{array}{l}\text { Serum and lesional skin } \\
\text { of vitiligo patients }\end{array}$ & MITF & miRNA microarray & 47 \\
\hline $\begin{array}{l}\text { MiRNA } \\
\text { polymorphisms }\end{array}$ & miR-196a-2 rs11614913 & - & Serum of vitiligo patients & TYR & $\begin{array}{l}\text { PCR genotyping and } \\
\text { RT-qPCR }\end{array}$ & 48,49 \\
\hline \multirow[t]{5}{*}{ Biomarkers } & $\begin{array}{l}\text { miR-1238-3p, } \\
\text { miR-202-3p, miR-630, } \\
\text { miR-766-3p }\end{array}$ & Downregulated & Serum of vitiligo patients & - & miRNA array & 50 \\
\hline & miR-16, miR-19b & Upregulated & Serum of NSV patients & - & miRNA array & 51 \\
\hline & miR-720 & Downregulated & Serum of NSV patients & - & miRNA array & 51 \\
\hline & miR-3940-5p & Downregulated & PBMC of NSV patients & - & miRNA array & 38 \\
\hline & $\begin{array}{l}\text { miR-4712-3p, } \\
\text { miR-224-3p }\end{array}$ & Upregulated & PBMC of NSV patients & - & miRNA array & 38 \\
\hline
\end{tabular}

NSV: nonsegmental vitiligo, IL: Interleukin, PBMC: peripheral blood mononuclear cell, T $\alpha$ 1: thymosin $\alpha 1$, MITF: microphthalmia-associated transcription factor, TYR: tyrosinase, HYAL: hyaluronidase, mirDIP: miRNA data integration portal, RT-qPCR: real-time quantitative PCR, SOCS1: suppressor of cytokine signaling 1, TRP1: tyrosinase-related protein 1, TGFBR2: transforming growth factor beta receptor 2, HO1: heme oxygenase 1, HSP: heat shock protein. 
vitiligo.

\section{MiRNAs associated with melanocyte proliferation, differentiation and melanogenesis}

It is most widely accepted that melanocyte destruction in vitiligo is caused by autoimmunity and oxidative stress-mediated toxicity. Thus, promoting the proliferation, migration and differentiation of melanocytes is an effective method for repigmentation of vitiligo. Research shows that multiple skin pigmentation disorders could potentially benefit from miRNA-based therapeutic applications, including vitiligo.

Microphthalmia-associated transcription factor (MITF), which is considered to be a master regulator of melanogenesis, upregulates the melanogenesis enzymes tyrosinase (TYR), tyrosinase-related protein 1 (TRP1) and TRP2 via binding to the M-box motif in their promoter regions. The findings showed that MITF deficiency can significantly influence the expression levels of some miRNAs. MITF knockdown in melanocytes induced upregulation of miR-1225-3p, miR634, miR-197, miR-766, miR-574-5p, and miR-328, however MITF knockdown downregulated the expression of miR-720 and miR-1308 ${ }^{39}$.

MiRNAs have been implicated in targeting TYR or MITF, which are potentially involved in the pigmentation process, to regulate their expression levels. For example, miR434-5p and miR-330-5p are upstream regulators of TYR expression, suggesting that these miRNAs could be used as potential therapeutic targets for pigmentation ${ }^{40,41,53}$. Other miRNAs, including miR-137, miR-148, and miR-145, were indicated by targeting MITF to mediate melanogenesis in vitiligo. Haflidadóttir et al. ${ }^{42}$ demonstrated miR-137 and miR-148 were shown to affect Mitf mRNA expression in melanoma cells through binding to $3^{\prime}$-UTR of mouse and human Mitf mRNA. miR-145 is a key regulator of many genes in the pigmentation process, such as TYR, TRP1, and $\mathrm{MITF}^{43}$. Overexpression of miR-145 reduces the expression of pigmentation genes such as Tyr, Myo5a and Rab27a. Furthermore, Šahmatova et al. ${ }^{44}$ determined the significant downregulation of miR-145 expression was detected in lesional skin of vitiligo patients.

Šahmatova et al. ${ }^{44}$ also indicated there is significant increased level of miR-155 in vitiligo lesional skin compared with the control subjects. In cell culture, it was shown that miR-155 contributed to the pathogenesis of vitiligo by downregulating multiple genes associated with melanogenesis, including suppressor of cytokine signaling 1 (SOCS1) and TRP1 and that increased levels of miR-155 contributed to development of the skin disease.

In addition, miRNAs were demonstrated to play an important role in regulating melanocyte differentiation, pig- mentation, proliferation, and cell survival. MiRNA-145 was demonstrated to be an important melanocyte proliferation-associated miRNA that may be involved in the initial inhibition of cell proliferation and induction of apoptosis, thereby playing an important role in disease initiation as well as the progression of vitiligo ${ }^{43}$.

Another miRNA, miR-211, may serve an important role in melanocyte biology and the development of vitiligo. As Dai et al. ${ }^{45}$ demonstrared miRNA-211 is highly expressed in primary melanocytes where it influences various cellular processes and positively regulates pigmentation by targeting transforming growth factor beta receptor 2 (TGFBR2). Loss of miRNA-211 is implicated in the stress response and melanomagenesis. Furthermore, the upregulation expression of miR-99b and miR-199a-3p were also detected in the skin of patients with vitiligo, and these miRNAs were found to have putative targets associated with melanocyte differentiation and melanogenesis ${ }^{44}$.

These results indicated the relevance of deregulated miRNAs as possible vitiligo markers and potential therapeutic targets.

\section{MiRNAs associated with oxidative stress in vitiligo}

The hypersensitivity induced by oxidative stress in cells plays a crucial role in determining melanocyte degeneration. Recently, several studies have found that the expression profile of miRNAs could be regulated by oxidative stress and could mediate the pathogenic effect of ROS in vitiligo. This suggests that miRNAs may participate in vitiligo pathogenesis by modulating the expression of oxidative stress related genes in melanocytes.

miR-1, miR-135a, miR-9, and miR-183 showed significant increase in expression in NSV skin compared to healthy $\operatorname{skin}^{37,54}$. Under oxidative stress, the increased expression of miR-1 expression increases has a potential role in the response to oxidative stress which favors apoptosis by targeting heat shock protein 60 (HSP60) and HSP70 mRNAs ${ }^{46,54}$. miR-183 was reported to target the $3^{\prime}$-UTR of heme oxygenase 1 (HO1) mRNA, which is a stress-responsive, antioxidant and anti-inflammatory factor ${ }^{37,55}$. miR-135a and miR-9 are known to target SIRT1 expression ${ }^{37}$. Several studies have indicated that SIRT1 protects against stress-related diseases by interacting with p53, forkhead box protein (FOXO), NF $\kappa \mathrm{B}$ and peroxisome proliferator-activated receptor gamma coactivator 1 -alpha (PGC-1 $\alpha$ ), which regulate diverse cellular processes, including stress responses and inflammation ${ }^{56,57}$.

In addition, the expression level of miR-25 increased in serum and lesion samples from vitiligo patients ${ }^{47}$. Research has demonstrated that oxidative the stress-induced increase in miR-25 in vitiligo plays a crucial role in promoting the degeneration of melanocytes by suppressing MITF 
in melanocytes ${ }^{47}$.

Therefore, the potential role of these miRNAs as drug targets for antioxidative therapy in vitiligo is worth investigating.

\section{MiRNA polymorphisms in vitiligo}

MiRNA polymorphisms may potentially interfere with miRNA-mediated regulation of cellular functions. SNPs are the most common genetic variants in the human genome, which influence the processing, expression, and targeted selection of miRNAs and play important roles in diverse physiological processes. A strong link has been demonstrated between miRNA SNPs and binding to mRNAs of genes involved in vitiligo susceptibility.

For example, individuals with TT and TC polymorphisms in the miR-196a-2 gene, combined with elevated levels of tyrosinase, have an increased risk of developing vitiligo as compared to those with CC genotype and normal tyrosinase levels. The CC genotype of miR-196a-2 rs11614913 is associated with decreased expression of tyrosinase-1 and correlates with lower levels of ROS. Thus, the $\mathrm{C}$ allele of miR-196a-2 protects against oxidative stress-mediated cell damage of melanocytes and diminishes the risk of developing vitiligo $^{48,49}$.

\section{Serum miRNA as potential biomarkers in vitiligo}

MiRNAs derived from melanocytes, immune cells and other related cells during vitiligo development might be detected in serum. Hence, circulating miRNAs may serve as novel biomarkers for diagnosis, monitoring therapy response and reflecting the disease severity of vitiligo.

Shang and $\mathrm{Li}^{50}$ determined that miR-1238-3p, miR-202-3p, miR-630 and miR-766-3p were significantly downregulated in the peripheral blood of vitiligo patients.

Another study showed that the expression levels of miR16 and miR-19b were upregulated, and miR-720 was downregulated in the serum of NSV patients ${ }^{51}$. Increased miR16 expression in peripheral blood mononuclear cells (PBMCs) was correlated with inflammation. miR-19b also plays a pivotal role in Th1 responses through facilitating IFN- $\gamma$ production and suppressing inducible regulatory T-cells ${ }^{58}$. In addition, Wang et al. ${ }^{38}$ also found that the expression of miR-3940-5p was decreased and miR-4712-3p and miR224-3p were significantly increased in the PBMCs of patients with NSV. Their analysis indicated that these differentially expressed miRNAs were associated with responses to the biological functions of $\mathrm{T} \alpha 1$, which has an important role in the mechanism of immune imbalance of vitiligo. Thus, these miRNAs appeared as potential serum biomarkers for distinguishing NSV from healthy controls.

These results suggest that circulating or serum miRNAs may be viable biomarker candidates for human vitiligo and that these miRNA-regulated gene networks are involved in oxidative stress and autoimmunity.

Although the role of miRNAs in regulating the pathogenesis of vitiligo has not yet been fully elucidated, the previous literatures have shown that miRNAs are closely related to the pathological process of vitiligo. These miRNAs are implicated in the process of abnormal melanocyte and pigment synthesis, inflammation and oxidative stress reactions, through targeting and regulating the key signaling proteins, which are involved in the pathological process of vitiligo. We have summary based on the existing reviews and research, and plotted a signal pathway networks shown as Fig. 2. Therefore, it is possible to apply these miRNAs as the therapeutic targets for vitiligo when discovering more novel miRNAs and elucidating their regulated signaling pathways.

\section{ADVANCES IN THERAPEUTIC APPLICATION OF VITILIGO}

\section{Common treatments for vitiligo}

\section{1) Immunomodulatory agents}

Systemic corticosteroids have been reported to rapidly arrest vitiligo spreading and to induce repigmentation. There are three common options for the treatment of vitiligo by systemic corticosteroids, including high-dose pulsed therapy, minipulsed therapy and daily oral low dose. Currently, the most common therapy is oral minipulse (OMP), which is a therapy in which moderate doses of betamethasone or dexamethasone are used. However, there is potential for side effects and the rate of relapses is high for this therapy, so the use of such an approach remains controversial. Topical corticosteroids are the most useful therapy for localized vitiligo, including topical steroids and calcineurin inhibitors, as they suppress immune responses in the superficial skin by decreasing immunoglobulins and complement levels. Halometasone is a synthetic, trihalogenated, topical glucocorticoid possessing anti-inflammatory properties. It has been reported to inhibit melanocyte destruction and regulate the local immune response by reducing the level of $\mathrm{IL}-17$ and increasing the level of $\mathrm{IL}-10^{59}$. Tacrolimus and pimecrolimus are used as topical calcineurin inhibitors and have also been introduced in the treatment of vitiligo currently ${ }^{60}$. They could prevent the activation of T-cells and the production of various inflammatory cytokines by inhibiting calcineurin action ${ }^{61}$.

\section{2) Phototherapy}

Phototherapy induces repigmentation through the mecha- 


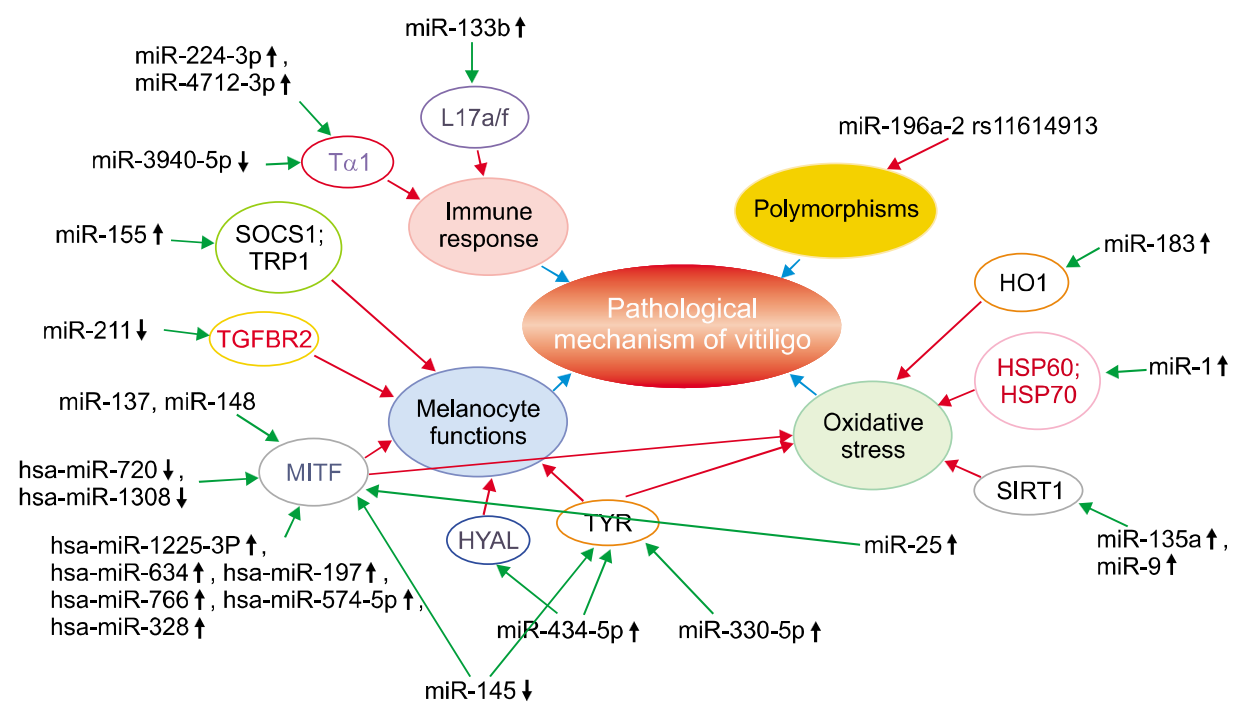

Fig. 2. MicroRNA (miRNAs) networks in vitiligo. MiRNAs regulate vitiligo pathology involved four aspects, inluding genetic polymorphisms, immune response, oxidative stress and melanocyte functions. Green arrows represent the effects of activating downstream target genes, and red arrows represent the effects of suppressing downstream target genes. T $\alpha$ 1: thymosin $\alpha 1$, MITF: microphthalmia-associated transcription factor, TYR: tyrosinase, HYAL: hyaluronidase, SOCS1: suppressor of cytokine signaling 1, TRP1: tyrosinase-related protein 1, TGFBR2: transforming growth factor beta receptor 2, HO1: heme oxygenase 1, HSP: heat shock protein.

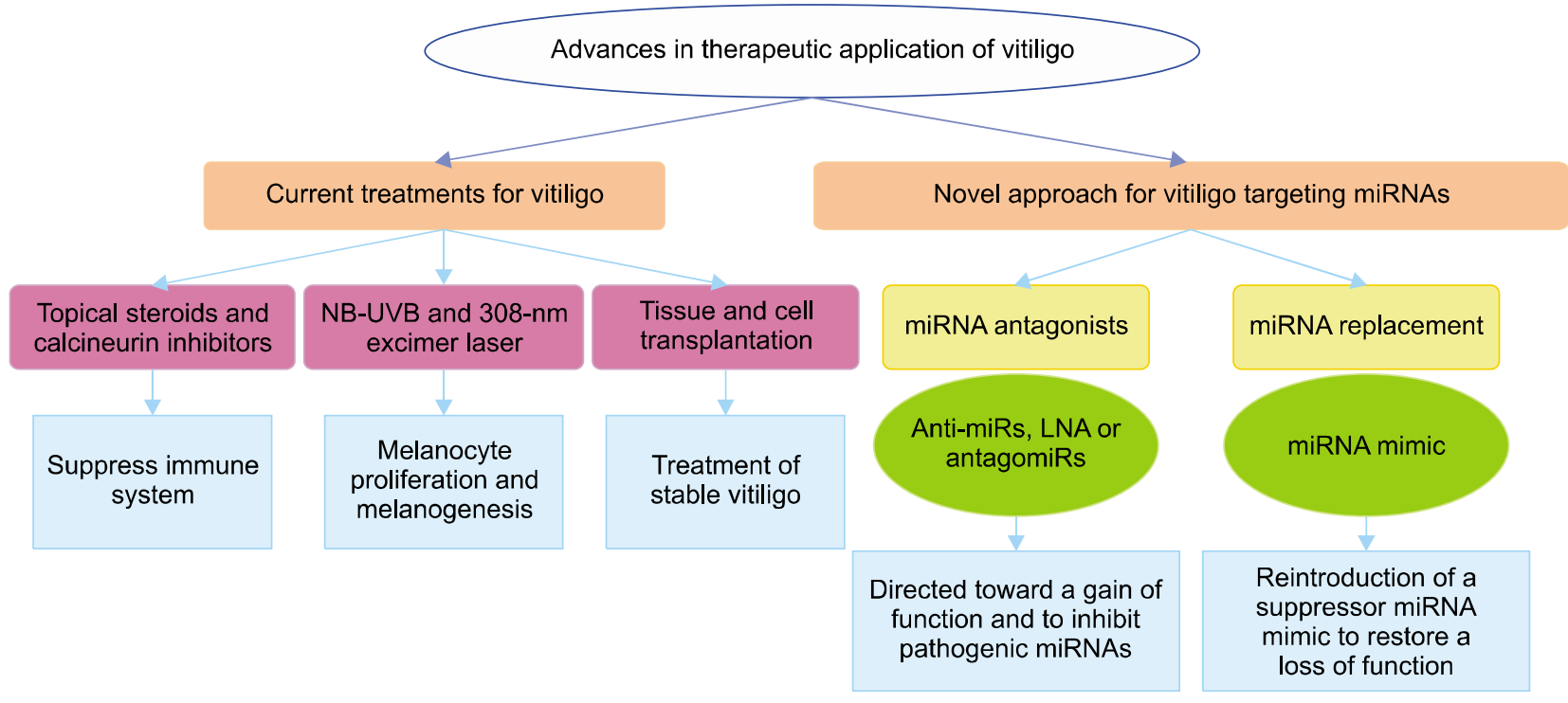

Fig. 3. The suggested treatments of vitiligo with advancing microRNA (miRNAs). Common treatments for vitiligo including immunomodulatory agents (topical or systemic), phototherapy and transplantation of pigment cells by surgical techniques. The novel application of miRNAs for vitiligo involved miRNA antagonists and miRNA replacement. NB-UVB: narrowband ultraviolet B, LNA: locked-nucleic acid.

nisms involving migration of melanocytes from perilesional skin, formation and melanization of melanosomes, and transfer of melanosomes into keratinocytes. Both photochemotherapy (PUVA) and ultraviolet B (UVB) phototherapy have been previously applied in vitiligo. However, PUVA therapy is less effective and associated with more adverse effects than narrowband UVB (NB-UVB) therapy; thus, it was rarely used at present ${ }^{62}$. The mechanism of NBUVB therapy in vitiligo involves inducing an increase in the release of melanocyte-stimulating growth factors by keratinocytes, which may promote proliferation of nearby melanocytes ${ }^{63}$. Furthermore, NB-UVB irradiation may directly enhance melanocyte migration, possibly through upregulating the expression level of phosphorylated focal 
adhesion kinase ( $\mathrm{p} 125 \mathrm{FAK})$ and matrix metalloproteinase2 (MMP2) in melanocytes ${ }^{64}$.

In addition, the recent use of the 308-nm excimer laser as a novel phototherapy was determined to be very effective for treating vitiligo ${ }^{65}$. Several studies demonstrated that the effectiveness and safety of the 308-nm excimer laser was superior to the other phototherapy, due to the advantage of having the ability to deliver higher energy fluences to the target tissue in less time, thereby stimulating a deeper reservoir of melanocytes in hair follicles. It can selectively treat a lesion while sparing the surrounding normal skin. Furthermore, a combination therapy with the 308-nm excimer laser and immunomodulatory agents (tacrolimus, pimecrolimus, or halometasone) has provided very motivating results, which may become a new treatment for vitiligo $^{66}$.

\section{3) Surgical techniques}

Surgical treatments are indicated for vitiligo patients who do not respond to medical treatments and whose vitiligo has been stable for at least two years. Several surgical techniques, including tissue and cell transplantation, have been proposed for the treatment of stable vitiligo. Various methods exist for tissue transplantation methods involving epidermal grafting, minigrafting and thin dermo-epidermal grafting.

However, cell therapy is an important alternative to conventional surgical methods in the treatment of pigmentary disorders with a large body involvement. A variety of cellular transplantation techniques have been investigated in vitiligo, including needling, melanocyte keratinocyte transplantations, split-thickness grafting, autologous punch grafting, and suction blister grafting ${ }^{67}$. Of the available surgical techniques, the melanocyte-keratinocyte transplantation procedure (MKTP) is an improved option that has gained popularity for the treatment of vitiligo patients ${ }^{68}$.

\section{Targeting miRNAs to treat vitiligo}

MiRNA-targeted treatments are perhaps a novel precision therapeutic as aberrantly expressed miRNAs in disease-affected cells have been widely reported. As more miRNAs have been identified as key players in the molecular pathogenesis of vitiligo, therapeutic applications based on miRNAs could potentially be beneficial for for multiple skin pigmentation disorders including vitiligo.

The therapeutic application of miRNAs involves two strategies (Fig. 3). One strategy is directed toward gain of function and aims to inhibit pathogenic miRNAs by using miRNA antagonists, such as anti-miRNAs, locked-nucleic acids (LNA), or antagomiRs. These miRNA antagonists are oligonucleotides with sequences complementary to the endogenous miRNA. They carry chemical modifications that enhance the affinity for the target miRNA and trap the endogenous miRNA in a configuration that is unable to be processed by RNA-induced silencing complex (RISC), or alternatively, lead to the degradation of the endogenous miRNA. Small molecule inhibitors specific for certain miRNAs are also being developed to inhibit miRNA function $^{69}$. For example, Maschmeyer et al. ${ }^{70}$ use sequence-specific cholesterol-modified oligonucleotides against miR-148a (antagomir-148a) for the selective elimination of proinflammatory Th1 cells in vivo. Antagomir-148a treatment could significant inhibit miR-148a expression and reduced the number of proinflammatory Th1 cells in the colon of colitic mice, indicated that antagomir-148a-mediated reduction of Th1 cells resulted in a significant amelioration of colitis. AntagomiRs of miR-155 could potentially assist in suppressing the activation of multiple inflammatory pathways and reducing the expression of proinflammatory cytokines by decreasing the effective levels of endogenous miR- $155^{71}$.

The second strategy, miRNA replacement, involves the reintroduction of a gene-suppressor miRNA mimic to modulate gene expression, often resulting in a loss of function effect. This approach essentially represents a second-generation RNAi-based therapy ${ }^{69}$. Su et al. ${ }^{72}$ demonstrated that the ability of melanocyte migration was altered by the upregulation of miR-211 using miR-211 mimic. Their results shown that the protein levels of MMP9 were significantly suppressed and the melanocyte migration was significantly reduced after transfection with the miR-211 mimic. Thus, miRNA mimic potentially represents an attractive therapeutic method to improve repigmentation outcomes in vitiligo patients.

Taken together, the therapeutic application of miRNAs has emerged as a highly promising therapeutic strategy. It encompasses several conceptual aspects of traditional gene therapy and technical features of siRNA therapeutics. However, given the fundamental differences in the approach, mechanism of action, and outcome, miRNA mimics should be viewed as a new class of therapeutics.

\section{CONFLICTS OF INTEREST}

The author has nothing to disclose.

\section{FUNDING SOURCE}

This work was supported by funding from National Natural Science Foundation of China (No. 81960562) and the GuangXi Natural Science Foundation (No. 2019GXNSFBA 185018). 


\section{DATA SHARING STATEMENT}

Research data are not shared.

\section{REFERENCES}

1. Malhotra N, Dytoc M. The pathogenesis of vitiligo. J Cutan Med Surg 2013;17:153-172.

2. Solak B, Dikicier BS, Cosansu NC, Erdem T. Effects of age of onset on disease characteristics in non-segmental vitiligo. Int J Dermatol 2017;56:341-345.

3. Bronevetsky Y, Ansel KM. Regulation of miRNA biogenesis and turnover in the immune system. Immunol Rev 2013; 253:304-316.

4. Yao Q, Song Z, Wang B, Zhang JA. Emerging roles of microRNAs in the metabolic control of immune cells. Cancer Lett 2018;433:10-17.

5. Spritz RA, Andersen GH. Genetics of vitiligo. Dermatol Clin 2017;35:245-255

6. Birlea SA, Ahmad FJ, Uddin RM, Ahmad S, Pal SS, Begum $\mathrm{R}$, et al. Association of generalized vitiligo with MHC class II loci in patients from the Indian subcontinent. J Invest Dermatol 2013;133:1369-1372.

7. Jin $Y$, Birlea SA, Fain PR, Mailloux CM, Riccardi SL, Gowan $\mathrm{K}$, et al. Common variants in FOXP1 are associated with generalized vitiligo. Nat Genet 2010;42:576-578.

8. Shi YL, Li K, Hamzavi I, Lim HW, Zhou L, Mi QS. Elevated circulating soluble interleukin-2 receptor in patients with non-segmental vitiligo in North American. J Dermatol Sci 2013;71:212-214.

9. Aly DG, Salem SA, Amr KS, El-Hamid MFA. A study of the association of glutathione S-transferase M1/T1 polymorphisms with susceptibility to vitiligo in Egyptian patients. An Bras Dermatol 2018;93:54-58.

10. Karam RA, Zidan HE, Khater $\mathrm{MH}$. Genetic variants of interferon-gamma and its mRNA expression and inflammatory parameters in the pathogenesis of vitiligo. Biochem Cell Biol 2017; 95:474-481.

11. Song P, Li K, Liu L, Wang X, Jian Z, Zhang W, et al. Genetic polymorphism of the Nrf2 promoter region is associated with vitiligo risk in Han Chinese populations. J Cell Mol Med 2016;20:1840-1850.

12. Jadeja SD, Mansuri MS, Singh M, Dwivedi M, Laddha NC, Begum R. A case-control study on association of proteasome subunit beta 8 (PSMB8) and transporter associated with antigen processing 1 (TAP1) polymorphisms and their transcript levels in vitiligo from Gujarat. PLoS One 2017;12:e0180958.

13. Grill C, Benzekri L, Rubod A, Aktary Z, Ezzedine K, Taïeb $A$, et al. Epidermal melanocytes in segmental vitiligo show altered expression of E-cadherin, but not P-cadherin. Br J Dermatol 2018;178:1204-1206.

14. Kim HJ, Uhm YK, Yun JY, Im SH, Yim SV, Chung JH, et al. Association between polymorphisms of discoidin domain receptor tyrosine kinase 1 (DDR1) and non-segmental vitiligo in the Korean population. Eur J Dermatol 2010;20:231-232.

15. Almasi-Nasrabadi M, Amoli MM, Robati RM, Rajabi F,
Ghalamkarpour F, Gauthier Y. CDH1 and DDR1 common variants confer risk to vitiligo and autoimmune comorbidities. Gene 2019;700:17-22.

16. Mansuri MS, Laddha NC, Dwivedi M, Patel D, Alex T, Singh $M$, et al. Genetic variations (Arg5Pro and Leu6Pro) modulate the structure and activity of GPX 1 and genetic risk for vitiligo. Exp Dermatol 2016;25:654-657.

17. Richmond JM, Frisoli ML, Harris JE. Innate immune mechanisms in vitiligo: danger from within. Curr Opin Immunol 2013;25:676-682.

18. Li S, Zhu G, Yang Y, Jian Z, Guo S, Dai W, et al. Oxidative stress drives CD8 + T-cell skin trafficking in patients with vitiligo through $\mathrm{CXCL} 16$ upregulation by activating the unfolded protein response in keratinocytes. J Allergy Clin Immunol 2017; 140:177-189.e9.

19. Li S, Zhu G, Yang Y, Guo S, Dai W, Wang G, et al. Oxidative stress-induced chemokine production mediates CD8(+) T cell skin trafficking in vitiligo. J Investig Dermatol Symp Proc 2015; 17:32-33.

20. Strassner JP, Harris JE. Understanding mechanisms of autoimmunity through translational research in vitiligo. Curr Opin Immunol 2016;43:81-88.

21. Cavalli G, Hayashi M, Jin Y, Yorgov D, Santorico SA, Holcomb C, et al. MHC class II super-enhancer increases surface expression of HLA-DR and HLA-DQ and affects cytokine production in autoimmune vitiligo. Proc Natl Acad Sci U S A 2016;113:1363-1368.

22. Dwivedi M, Laddha NC, Shah K, Shah BJ, Begum R. Involvement of interferon-gamma genetic variants and intercellular adhesion molecule-1 in onset and progression of generalized vitiligo. J Interferon Cytokine Res 2013;33:646659.

23. Miniati A, Weng Z, Zhang B, Therianou A, Vasiadi M, Nicolaidou $E$, et al. Stimulated human melanocytes express and release interleukin-8, which is inhibited by luteolin: relevance to early vitiligo. Clin Exp Dermatol 2014;39:54-57.

24. Ashour AE, Ahmed AF, Kumar A, Zoheir KM, Aboul-Soud MA, Ahmad SF, et al. Thymoquinone inhibits growth of human medulloblastoma cells by inducing oxidative stress and caspase-dependent apoptosis while suppressing NF-kB signaling and IL-8 expression. Mol Cell Biochem 2016;416: 141-155.

25. Iannella G, Greco A, Didona D, Didona B, Granata G, Manno A, et al. Vitiligo: pathogenesis, clinical variants and treatment approaches. Autoimmun Rev 2016;15:335-343.

26. Klarquist J, Eby JM, Henning SW, Li M, Wainwright DA, Westerhof $W$, et al. Functional cloning of a gp100-reactive T-cell receptor from vitiligo patient skin. Pigment Cell Melanoma Res 2016;29:379-384.

27. Kovacs D, Abdel-Raouf $\mathrm{H}$, Al-Khayyat $M$, Abdel-Azeem $E$, Hanna MR, Cota $C$, et al. Vitiligo: characterization of melanocytes in repigmented skin after punch grafting. J Eur Acad Dermatol Venereol 2015;29:581-590.

28. Di Meglio P, Duarte JH. CD8 T Cells and IFN-y emerge as critical players for psoriasis in a novel model of mouse psoriasiform skin inflammation. J Invest Dermatol 2013;133: 871-874. 
29. Jian Z, Li K, Song P, Zhu G, Zhu L, Cui T, et al. Impaired activation of the Nrf2-ARE signaling pathway undermines $\mathrm{H} 2 \mathrm{O} 2$-induced oxidative stress response: a possible mechanism for melanocyte degeneration in vitiligo. J Invest Dermatol 2014;134:2221-2230.

30. Thannickal VJ, Fanburg BL. Reactive oxygen species in cell signaling. Am J Physiol Lung Cell Mol Physiol 2000;279: L1005-L1028.

31. Xie H, Zhou F, Liu L, Zhu G, Li Q, Li C, et al. Vitiligo: how do oxidative stress-induced autoantigens trigger autoimmunity? J Dermatol Sci 2016;81:3-9.

32. Manucha W. [Mitochondria and oxidative stress participation in renal inflammatory process]. Medicina (B Aires) 2014;74: 254-258. Spanish.

33. Yi X, Guo W, Shi Q, Yang Y, Zhang W, Chen X, et al. SIRT3-dependent mitochondrial dynamics remodeling contributes to oxidative stress-induced melanocyte degeneration in vitiligo. Theranostics 2019;9:1614-1633.

34. Mohammed GF, Gomaa AH, Al-Dhubaibi MS. Highlights in pathogenesis of vitiligo. World J Clin Cases 2015;3:221-230.

35. Basnet B, Bhushan A, Khan R, Kumar G, Sharma VK, Sharma $A$, et al. Plasma \& urinary catecholamines \& urinary vanillylmandelic acid levels in patients with generalized vitiligo. Indian J Med Res 2018;147:384-390.

36. Sugiura $S$, Tazuke $M$, Ueno $S$, Sugiura $Y$, Kato I, Miyahira $Y$, et al. Effect of prolactin-induced protein on human skin: new insight into the digestive action of this aspartic peptidase on the stratum corneum and its induction of keratinocyte proliferation. J Invest Dermatol 2015;135:776785.

37. Mansuri MS, Singh M, Dwivedi M, Laddha NC, Marfatia YS, Begum R. MicroRNA profiling reveals differentially expressed microRNA signatures from the skin of patients with nonsegmental vitiligo. Br J Dermatol 2014;171:1263-1267.

38. Wang $\mathrm{Y}$, Wang $\mathrm{K}$, Liang J, Yang $\mathrm{H}$, Dang $\mathrm{N}$, Yang $\mathrm{X}$, et al. Differential expression analysis of miRNA in peripheral blood mononuclear cells of patients with non-segmental vitiligo. J Dermatol 2015;42:193-197.

39. Wang $\mathrm{P}$, Li Y, Hong W, Zhen J, Ren J, Li Z, et al. The changes of microRNA expression profiles and tyrosinase related proteins in MITF knocked down melanocytes. Mol Biosyst 2012;8:2924-2931.

40. Wu DTs, Chen JS, Chang DC, Lin SL. Mir-434-5p mediates skin whitening and lightening. Clin Cosmet Investig Dermatol 2008;1:19-35.

41. Rambow F, Bechadergue A, Saintigny G, Morizot F, Mahé C, Larue L. miR-330-5p targets tyrosinase and induces depigmentation. J Invest Dermatol 2014;134:2846-2849.

42. Haflidadóttir BS, Bergsteinsdóttir K, Praetorius C, Steingrímsson E. miR-148 regulates Mitf in melanoma cells. PLoS One 2010;5:e11574.

43. Dynoodt P, Mestdagh P, Van Peer G, Vandesompele J, Goossens K, Peelman LJ, et al. Identification of miR-145 as a key regulator of the pigmentary process. J Invest Dermatol 2013;133:201-209.

44. Šahmatova L, Tankov S, Prans E, Aab A, Hermann H, Reemann $\mathrm{P}$, et al. MicroRNA-155 is dysregulated in the skin of patients with vitiligo and inhibits melanogenesis-associated genes in melanocytes and keratinocytes. Acta Derm Venereol 2016;96:742-747.

45. Dai X, Rao C, Li H, Chen Y, Fan L, Geng H, et al. Regulation of pigmentation by microRNAs: MITF-dependent microRNA-211 targets TGF- $\beta$ receptor 2. Pigment Cell Melanoma Res 2015;28:217-222.

46. Xu C, Lu Y, Pan Z, Chu W, Luo X, Lin H, et al. The muscle-specific microRNAs miR-1 and miR-133 produce opposing effects on apoptosis by targeting HSP60, HSP70 and caspase-9 in cardiomyocytes. J Cell Sci 2007;120(Pt 17):3045-3052.

47. Shi Q, Zhang W, Guo S, Jian Z, Li S, Li K, et al. Oxidative stress-induced overexpression of miR-25: the mechanism underlying the degeneration of melanocytes in vitiligo. Cell Death Differ 2016;23:496-508.

48. Cui TT, Yi XL, Zhang WG, Wei C, Zhou FB, Jian Z, et al. miR-196a-2 rs11614913 polymorphism is associated with vitiligo by affecting heterodimeric molecular complexes of Tyr and Tyrp1. Arch Dermatol Res 2015;307:683-692.

49. Huang $Y$, Yi X, Jian Z, Wei C, Li S, Cai C, et al. A single-nucleotide polymorphism of miR-196a-2 and vitiligo: an association study and functional analysis in a Han Chinese population. Pigment Cell Melanoma Res 2013;26: 338-347.

50. Shang Z, Li H. Altered expression of four miRNA (miR1238-3p, miR-202-3p, miR-630 and miR-766-3p) and their potential targets in peripheral blood from vitiligo patients. J Dermatol 2017; 44:1138-1144.

51. Shi YL, Weiland M, Li J, Hamzavi I, Henderson M, Huggins $\mathrm{RH}$, et al. MicroRNA expression profiling identifies potential serum biomarkers for non-segmental vitiligo. Pigment Cell Melanoma Res 2013;26:418-421.

52. Wang Y, Wang K, Dang N, Wang L, Zhang M. Downregulation of miR-3940-5p promotes T-cell activity by targeting the cytokine receptor IL-2R gamma on human cutaneous T-cell lines. Immunobiology 2016;221:1378-1381.

53. Mione $M$, Bosserhoff $A$. MicroRNAs in melanocyte and melanoma biology. Pigment Cell Melanoma Res 2015;28: 340-354.

54. Mansuri MS, Singh M, Begum R. miRNA signatures and transcriptional regulation of their target genes in vitiligo. J Dermatol Sci 2016;84:50-58.

55. Ke K, Sul OJ, Rajasekaran M, Choi HS. MicroRNA-183 increases osteoclastogenesis by repressing heme oxygenase-1. Bone 2015;81:237-246.

56. Wu Q, Shi M, Meng W, Wang Y, Hui P, Ma J. Long noncoding RNA FOXD3-AS1 promotes colon adenocarcinoma progression and functions as a competing endogenous RNA to regulate SIRT1 by sponging miR-135a-5p. J Cell Physiol 2019;234:21889-21902.

57. Saunders LR, Sharma AD, Tawney J, Nakagawa M, Okita K, Yamanaka S, et al. miRNAs regulate SIRT1 expression during mouse embryonic stem cell differentiation and in adult mouse tissues. Aging (Albany NY) 2010;2:415-431.

58. Jiang S, Li C, Olive V, Lykken E, Feng F, Sevilla J, et al. Molecular dissection of the miR-17-92 cluster's critical dual 
roles in promoting Th1 responses and preventing inducible Treg differentiation. Blood 2011;118:5487-5497.

59. The Execare Working Group. Halometasone $0.05 \%$ cream in eczematous dermatoses. J Clin Aesthet Dermatol 2013;6: 39-44.

60. Wong R, Lin AN. Efficacy of topical calcineurin inhibitors in vitiligo. Int J Dermatol 2013;52:491-496.

61. Sisti A, Sisti G, Oranges CM. Effectiveness and safety of topical tacrolimus monotherapy for repigmentation in vitiligo: a comprehensive literature review. An Bras Dermatol 2016; 91:187-195.

62. Esmat S, Hegazy RA, Shalaby S, Hu SC, Lan CE. Phototherapy and combination therapies for vitiligo. Dermatol Clin 2017;35:171-192.

63. Shafiee A, Hoormand M, Shahidi-Dadras M, Abadi A. The effect of topical piperine combined with narrowband UVB on vitiligo treatment: a clinical trial study. Phytother Res 2018;32:1812-1817.

64. Fawzy MM, El Maadawi ZM, Hegazy RA, El Fatah NS. Vitiligo- The story from within: a transmission electron microscopic study before and after narrow-band ultraviolet B. Ultrastruct Pathol 2016;40:265-275.

65. Fa $Y$, Lin $Y$, Chi XJ, Shi $W H$, Wang JL, Guo $X$, et al. Treatment of vitiligo with 308-nm excimer laser: our experience from a 2-year follow-up of 979 Chinese patients. J Eur Acad Dermatol Venereol 2017;31:337-340.

66. Wang LM, Lu WJ, Yuan JT, Zeng BB, Li D, Zhang F, et al.
Utility of dermoscopy for evaluating the therapeutic efficacy of tacrolimus ointment plus 308-nm excimer laser combination therapy in localized vitiligo patients. Exp Ther Med 2018;15:3981-3988.

67. Dillon $A B$, Sideris A, Hadi A, Elbuluk N. Advances in vitiligo: an update on medical and surgical treatments. J Clin Aesthet Dermatol 2017; 10:15-28.

68. Nahhas AF, Mohammad TF, Hamzavi IH. Vitiligo surgery: shuffling melanocytes. J Investig Dermatol Symp Proc 2017; 18:S34-S37.

69. Gambari R, Brognara E, Spandidos DA, Fabbri E. Targeting oncomiRNAs and mimicking tumor suppressor miRNAs: new trends in the development of miRNA therapeutic strategies in oncology (Review). Int J Oncol 2016;49:5-32.

70. Maschmeyer P, Petkau G, Siracusa F, Zimmermann J, Zügel F, Kühl AA, et al. Selective targeting of pro-inflammatory Th1 cells by microRNA-148a-specific antagomirs in vivo. J Autoimmun 2018;89:41-52.

71. Zhou S, Wang Y, Meng Y, Xiao C, Liu Z, Brohawn P, et al. In vivo therapeutic success of microRNA-155 antagomir in a mouse model of lupus alveolar hemorrhage. Arthritis Rheumatol 2016;68:953-964.

72. Su M, Miao F, Jiang S, Shi Y, Luo L, He X, et al. Role of the p53-TRPM1/miR-211-MMP9 axis in UVB-induced human melanocyte migration and its potential in repigmentation. Int J Mol Med 2020;45:1017-1026. 